\title{
Philosophical Thinking of Emotional Calculation
}

\author{
Xiao-meng ZHANG \\ Information Center of Beijing Institute of Fashion \\ Technology \\ Beijing, 100029, China \\ 2540944916@qq.com
}

\author{
Hai-bo ZHANG \\ Library of Beijing Institute of Fashion \\ Technology \\ Beijing, 100029, China \\ hbdmzhb@126.com
}

\begin{abstract}
Emotional calculation, one of the heated topics in artificial intelligent research, is widely applied. But at the same time, there are a series of problems. In order to reduce the issues arising in the development of artificial intelligence in the field of artificial intelligence and to develop emotional artificial intelligence in the direction of health, this paper studies emotional calculation from the perspective of philosophical thinking, and tells the importance of philosophical thinking and the influence of emotional computing on society. Taking the emotional calculation of the male $T$ shirt image as an example, this paper tells the realization of the philosophical theory guiding the emotional calculation of the male $T$-shirt clothing image, in order to demonstrate the healthy direction of the emotional artificial intelligence can not be separated from the philosophical thinking. The exploration is advantageous to provide a reference for the study of emotional calculation to the experts and scholars, and to provide ideas for the philosophical theory of emotional artificial intelligence to realize the real emotional interaction between mankind and machines.
\end{abstract}

Keywords-Emotional calculation; Philosophical thinking; Healthy direction; Clothing image; Emotional interaction

\section{INTRODUCTION}

In 1985, M.Minsky, one of the founders of artificial intelligence, proposed that "the key problem of machine intelligence is whether the computer can achieve intelligence without emotion"[1]. The theoretical research of artificial intelligence is aimed at how the computer system can accomplish the task under the support of human wisdom. Its theory is based on a very abstract philosophical thinking, and the essence is to simulate mode of human thinking. In social activities, human activity is mainly the communication between person, such as the information of the image itself, communication will produce a lot of information, and most of the information produced is emotional information. From the perspective of cognitive science, emotion analysis belongs to the level of advanced intelligence, and affective computing technology is humanized human-machine interaction. Emotion computing technology, human intelligence, perception, cognition and expression of emotional capabilities be endowed with the computer system, thereby relying on human intelligence to achieve human-computer interaction. The process of emotion calculation includes emotion signal acquisition, emotional semantic analysis recognition and emotion understanding. It is initiated by philosophy concept, and exists in the way of multidisciplinary integration and technology. It is an innovation in the field of artificial intelligence now. It plays a great role in promoting the development of the new era of human society.

The research of affective computing technology is multidisciplinary. The key point is to collect emotional characteristic information, get emotional computing model and establish emotional semantic space. Nowadays, more and more "emotional" products emerge in the field of artificial intelligence, which has a profound impact on mankind and the future. However, while enjoying the development of artificial intelligence, we should also think about the relationship between emotional calculation and human intelligence from a philosophical point of view, and carry out deep philosophical thinking on emotional calculation, so that we can better guide human practice and create the greater value for the development of human and society and promote emotion calculation. Finally, it promotes the development of affective computing technology and philosophy.

\section{THE NECCESSITY OF PHILOSOPHICAL THINKING}

It is that starting from reality, seeking truth from facts, and causing qualitative change by quantitative change, these two concepts are important viewpoints in philosophy and the key theory to guide human practice. Emotional computing technology is the result of mutual promotion and development between philosophy and science. Emotional computing technology, on the one hand, is the demand for the continuous development of artificial intelligence, and on the other hand, it is the requirement of humanized human-machine interaction. Emotional intelligence machines can simulate artificial life to obtain emotional ability, and integrate with logical computing to achieve real artificial intelligence. It is qualitative progress in the framework of artificial intelligence. Emotional computing is one of the most important research directions in the field of artificial intelligence. It can interact with human emotions, and has ability of emotional connectivity, then to satisfy people's emotional and psychological needs, so that we can gradually build trust and dependence between computer and humanity to realize "humanization" interaction. Emotional computing can provide users with better services and satisfy users' emotional needs under philosophical thinking. 
Since the research of AI has attracted much attention from researchers in the field of science, its research is relatively limited and faces challenges. For example, emotional information is easily affected by the factors such as environment, physiology, psychology, cultures, backgrounds, contexts, and semantics. It is also a difficult point whether the emotional features can be extracted and calculated accurately in emotional computing, and many methods have its application conditions. The research of emotional computing technology requires to solve the problem that needs philosophers and scientific researchers to make a lot of efforts. In the face of the complex emotion of human beings and the problem that human beings do not understand, emotional artificial intelligence still has a long way to go [2].

\section{RELATIONSHIP BETWEEN EMOTIONAL COMPUTING AND PHILOSOPHY}

Philosophy stresses materialist conception of history, focuses on the cognition of things, and then pays close attention to and explores the objective material world. Similarly, philosophy is concerned about human emotional intelligence and cares about emotional AI. Philosophy has provided theoretical guidance for the development of emotional AI, and intelligent technology has opened up human thinking and promoted the development of philosophy. Everything is developing, what technology brings innovation, and the progress of technology has promoted the development of history. Affective computing is closely related to Philosophy in the field of AI. It is a science that produced by human beings understanding the world and transforming the world in the process of.

The emotional expression of human beings is subjective. Emotional calculation is the emotional expression of emotion, which makes the computer think as scientifically as human, effectively simulates human thinking and recognizes the essential features of things. Emotional computing technology collects and analyzes emotional information, and makes a deeper study and understanding of the essence of intelligence, which promotes the development of the interdisciplinary between philosophy and science. Philosophy is the first to think about the problem, to clarify the basic concepts, and to find the confluence point, the delineation of the field, the analysis and evaluation, the Critical Argumentation in the research results of different disciplines. In the research of affective computing in a certain field, scholars first think about the idea and work of implementation, including the application method. Thus to clarify the basic principles of the interdisciplinary in the emotional calculation, clarify the basic concepts, design the methods and procedures of emotional calculation, and demonstrate the feasibility with the relevant theory, which embodies the development of intelligence without the participation of philosophy.

The role of philosophy is how to transition from the existing mental semantic processing to the level of machine realization. From a philosophical point of view, philosophy has been applied to the research field of affective computing technology, so that philosophy is an indispensable part of scientific development. In the study of emotional computing, we should always have a clear mind to understand the essence of the problem, to solve the problem in a scientific way and to treat the problem with a rigorous philosophical spirit [3-4].

\section{THE IMPACT OF THE DEVELOPMENT OF EMOTIONAL COMPUTING ON HUMAN SOCIETY}

Emotional calculation has made great contributions to the development of human society. It provides the possibility of sharing labor for human beings, not only physical labor, but also the function of reading people's thinking. However, there are many problems. These problems have become the hot spots of people's attention, which has led to philosophical thinking and debate.

The development of emotional computing technology has sprung up many research results and "emotionally" products. It has brought economic benefits to human beings, promoted social progress, impacted the traditional ideas of human beings, liberated people's psychological world, greatly expanded human physical strength and awareness, and better established the point of new views of philosophers. But the development of emotional intelligent technology has also made separate and no longer engaged in simple labor as for some workers, so they have to change their way of work. When person studies the new field in practice, it is urgent that it can replace a lot of unnecessary and complicated labor, especially emotional artificial intelligence. Human beings are eager to replace the labor of human emotion analysis, but it will also worry about the development that it will cause new social problems.

In general, the development of affective computing technology promotes the progress of science and technology, promotes the continuous improvement of human knowledge, and has a profound and extensive impact on mankind.

\section{THE APPLICATION OF EMOTIONAL CALCULATION IN THE PHILOSOPHICAL PERSPECTIVE}

The key to philosophy lies in thinking and analyzing problems objectively. In the research of affective computing technology, emotion analysis is needed to consider how to achieve the purpose of research. For sentiment analyses, there are two main types of analytical methods: one is based on the dictionary method, relying on the rules and dictionaries, and using syntactic analyses text to calculate their emotional values, and to describe the essential features of the images according to the statistical emotional values, such as the e-commerce website, the evaluation of the network and so on [5]. The two is based on the machine learning method, such as image recognition, speech recognition, human emotion recognition, emotional semantic analysis, quantitative low layer feature extraction, support vector machine training, model building, large scene virtual reality combined with multi mode dialogue simulation research. The test data is built to model the results of emotional calculation.

For example, the emotional computing of Zhang and other, that emotional analysis of clothing images is based on machine learning method to analyze affective. First, teachers and students are asked to collect emotional words. After consulting the information and seeking advice from the experts in the field of clothing, the emotional words in the opposite direction are 
finally determined, "Simple - Gorgeous", "Steady - Sports", "Formal - Leisure", "Plain - Fashion", "Classic - Modern", "Elegant - Rough" and so on. Then these images are graded on the Web Page Emotion Test System according to emotion words. Finally, the emotional words value of the image of clothing is counted in the background, which that the emotional calculation of the clothing image is realized [6-7], as shown in Figure 1 and Figure 2. After statistics of emotional word values, multivariate statistical factor analysis method is used to reduce the dimension of variables and select a few factors with the higher power of expression. After the analysis, such as the maximum difference rotation method and the principal component analysis method, the factor load matrix and factor score function coefficient matrix is established, and the emotional semantic space of the clothing image is finally established.

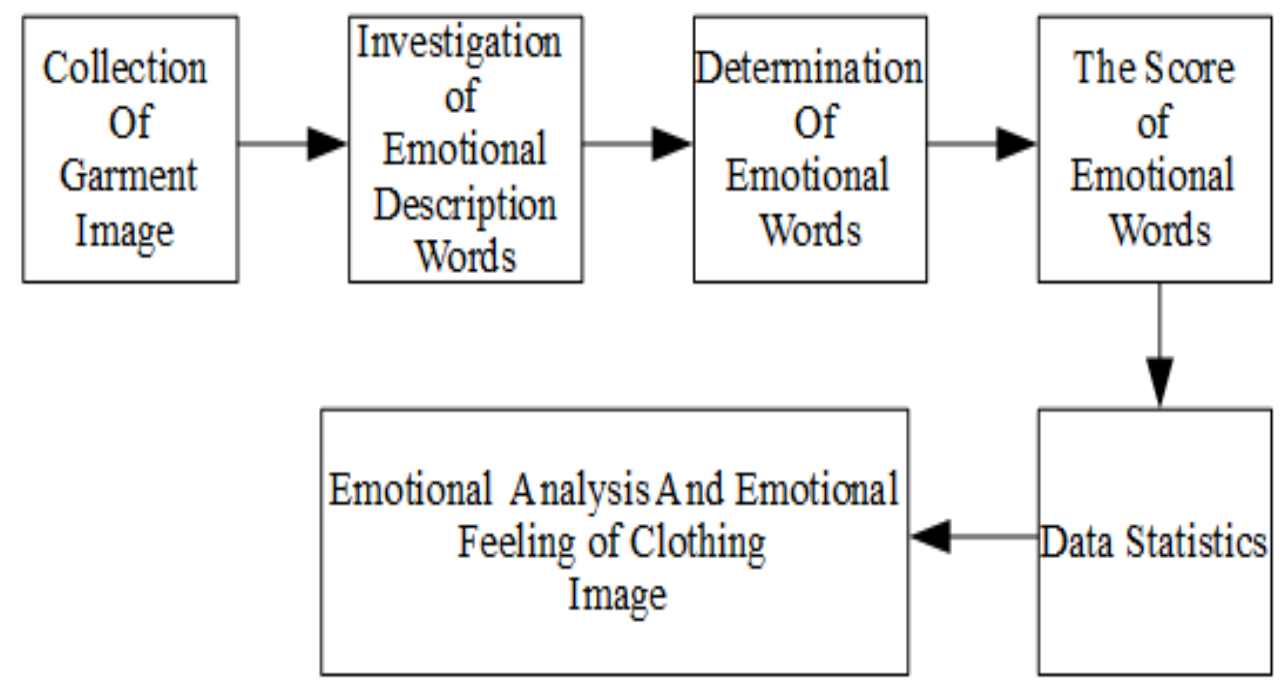

Fig 1. Research framework of clothing emotional computing

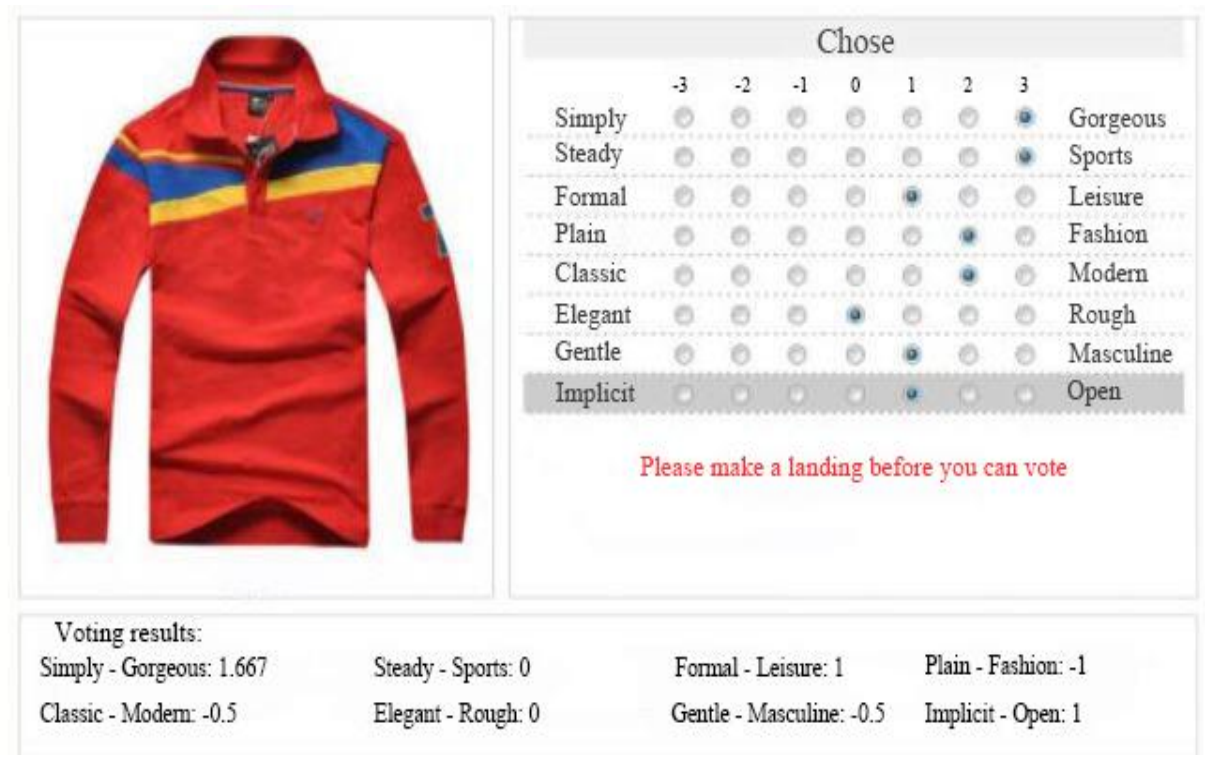

Fig 2. The score of emotional words

In the research of emotional calculate of male T-shirt clothing image, the first step is to score the emotion word and establish the emotional semantic space. Furthermore, the feature extraction of the image is extracted. The extracted factors are mapped to the emotional semantic space so that the emotional factor values are calculated. Finally, the image of the man T-shirt clothing is retrieved according to the similarity of the emotional factor value. In the process of research, selecting the appropriate method requires exploring the principle of algorithms through philosophical thinking. And quite a few experiments have been carried out to reduce the problems in the research of male $\mathrm{T}$-shirt clothing image emotional computing so that to achieve the desired results of the experiment. 
From a philosophical point of view, the emotional computation of clothing images correctly looks at the nature of the problem. According to the purpose of the study, the theory and method can be realized. So it seems that, it is of great significance to guide the theory of philosophy to simulate the behavior of human emotional intelligence and to realize calculation of emotion in the philosophical sense.

\section{CONCLUSION}

The development of the field of emotional AI needs further breakthroughs, which is what we expect. This article explores the philosophical thinking of affective computing from the perspective of the relationship between emotional computing and philosophy, the application of affective computing in social life and its impact on society. There is still a lot of controversy in the theoretical circle of emotional artificial intelligence, but it also shows that the research of emotional computing is arduous, and that emotional computing is an important direction of the development of artificial intelligence.

The development and application of emotional computing in the field of artificial intelligence is very broad. The simulation of human emotional intelligence can not only bring social and economic benefits, promote the development of science and technology and the progress of human thought, but also fully reflect the improvement of perception and cognitive intelligence. But in the realization of emotional interaction with human beings, it is still necessary to take a philosophical reflection and treat it seriously so as to adapt to the pace of social development and develop healthier.

\section{ACKNOWLEDGMENT}

The work described in this article was supported by grants from Technology General Project of Beijing Municipal Commission of Education (No.AJ2016-11).

\section{REFERENCES}

[1] Song Miao-ye, The Philosophy Study of Affective Computing [D], Dong Hua University, October, 2010. (In Chinese)

[2] Guo Teng-fei, Philosophical Thinking of Artificial Intelligence[D], Shan Xi University, June, 2011. (In Chinese)

[3] Li Fei-fei, The Next Spring of Artificial Intelligence is Emotional and Emotional Computing [EB/OL], http://www.sohu.com/, January, 2018. (In Chinese)

[4] Xu Ying-jin, Why does the research of artificial intelligence require philosophical participation [EB/OL], http://www.sohu.com/, October, 2017. (In Chinese)

[5] Huang Su-yu, Application of LDA model based on sentiment analysis in online reviews $[J]$, http://kns.cnki.net/kcms/detail/22.1256.F.20180319.1053.118.html, March, 2018, 82-83. (In Chinese)

[6] Zhang Hai-bo, Huang Tie-jun, Wan Fei-fei, Clothing Fabric Image Emotion Testing System Based on Web [J], Knitting Industries, 2012(08): 55-58. (In Chinese)

[7] Zhang Hai-bo, Liu Rui-pu, Cheng Wen-gang, Wang Yi, Li Xiao-ling, A Preliminary Study of Clothing Emotion Calculation[J], Knitting Industries, 2008(08): 39-41. (In Chinese) 